\title{
Discovering cosmic rays with OCRA: outreach activities for students and teachers
}

\section{S. Hemmer ${ }^{a^{*}}$, C. Aramo ${ }^{b}$, E. Bissaldi ${ }^{c, d}$, V. Bocci ${ }^{e}$, B. Bottino ${ }^{f, g}$, M. Buscemi $^{h}$, L. Caccianiga $^{i}$, G. Cataldi ${ }^{\prime}$, F. Dimiccoli ${ }^{k}$, F. Di Pierro ${ }^{l}$, C. Evoli ${ }^{m, n}$, A. Giampaoli ${ }^{m}$, G. La Vacca $^{o, p}$, A. Menegolli ${ }^{q, r}$, S. Miozzi ${ }^{i}$, F. Morsani ${ }^{t}$, R. Munini ${ }^{u, v}$, R. Paoletti ${ }^{t}$, D. \\ Rozza $^{w, x}$, M. Schioppa ${ }^{y, z}$, N. Tomassetti ${ }^{a a, b b}$ and E. Vannuccini ${ }^{c c}$ on behalf of the OCRA Collaboration}

(a complete list of authors can be found at the end of the proceedings)

$a_{\text {INFN Sezione di Padova, Padua, Italy }}$

$b_{\text {INFN Sezione di Napoli, Naples, Italy }}$

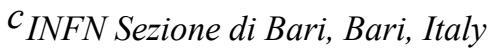

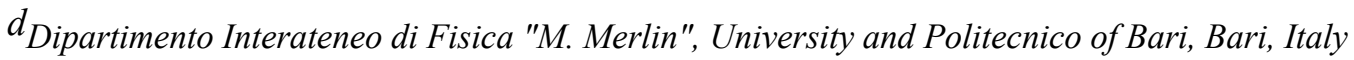

$e_{\text {INFN Sezione di Roma I, Rome, Italy }}$

$f_{\text {INFN Sezione di Genova, Genua, Italy }}$

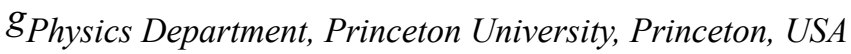

$h_{\text {INFN Sezione di Catania, Catania, Italy }}$

${ }^{i}$ INFN Sezione di Milano, Milan, Italy

$j$ INFN Sezione di Lecce, Lecce, Italy

$k_{\text {Trento Institute for Fundamental Physics and Applications, Trento, Italy }}$

$l_{\text {INFN Sezione di Torino, Turin, Italy }}$

m INFN Laboratori Nazionali del Gran Sasso, Assergi, Italy

${ }^{n}$ Gran Sasso Science Institute, L'Aquila, Italy

${ }^{o}$ INFN Sezione di Milano Bicocca, Milan, Italy

pipartimento di Fisica "Giuseppe Occhialini", University of Milan-Bicocca, Milan, Italy

*Speaker 
$q_{\text {INFN Sezione di Pavia, Pavia, Italy }}$

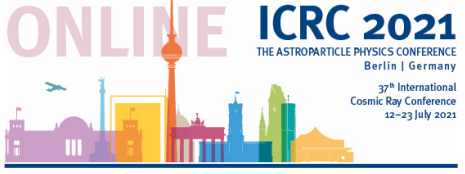

${ }^{r}$ Dipartimento di Fisica, University of Pavia, Pavia, Italy

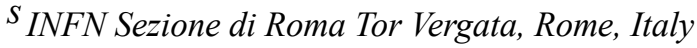

${ }^{t}$ INFN Sezione di Pisa, Pisa, Italy

$u_{\text {INFN Sezione di Trieste, Trieste, Italy }}$

$v$ Institute for Fundamental Physics of the Universe, Trieste, Italy

w INFN Laboratori Nazionali del Sud, Catania, Italy

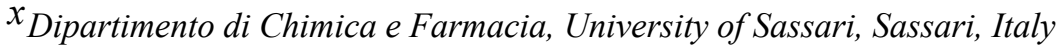

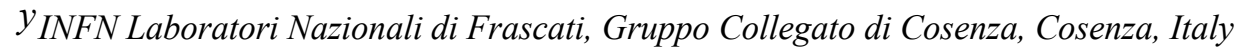

${ }^{z}$ Dipartimento di Fisica, University of Calabria, Arcavacata di Rende, Italy

a INFN Sezione di Perugia, Perugia, Italy

bb Dipartimento di Fisica e Geologia, University of Perugia, Perugia, Italy

cc INFN Sezione di Firenze, Florence, Italy

E-mail: sabine.hemmer@pd.infn.it

The Outreach Cosmic Ray Activities (OCRA) project offers a platform for all outreach activities focusing on cosmic rays within the Italian Istituto Nazionale di Fisica Nucleare (INFN). Created in 2018, OCRA now counts 21 of the institute's divisions all over Italy as members. The project's core activity is the participation of all its members in the International Cosmic Day, organized by DESY, and inviting high school students to carry out hands-on measurements of the cosmic ray flux and learn about the related physics background. Two students from each division are then selected to participate in the annual OCRA science camp, a three-day full immersion into the life of a physicist. The national activities are complemented by local initiatives of the OCRA member groups: workshops and tutorship programs, science competitions and the development of new detectors for outreach activities offer a multitude of possibilities for students to engage with our researchers and to explore the world of cosmic rays. Since spring of 2020 OCRA also offers a series of online laboratories on its website https://web.infn.it/OCRA/, designed not only to be used by students individually but also to be used in the classroom by teachers. Here we will give an overview on all activities offered by OCRA with a particular focus on the 2020 online event organized in occasion of the International Cosmic Day, that saw the participation of more than 3000 students.

37th International Cosmic Ray Conference (ICRC 2021)

July 12th - 23rd, 2021

Online-Berlin, Germany 


\section{Introduction}

OCRA - Outreach Cosmic Ray Activities was born in 2018 as a national outreach project of the Istituto Nazionale di Fisica Nucleare (INFN). The project was created with the aim of building a national framework for the institute's numerous public engagement activities in the field of cosmic ray physics already present at a local level in its divisions and national laboratories. Currently 21 INFN divisions and laboratories, located all over Italy, participate in the project.

Just like the number of participants, also the number of activities that are developed and carried out within the OCRA project has been growing larger and larger over the past years. They range from activities coordinated on a national level, like the yearly participation to the International Cosmic Day organized by DESY [1] and the organization of a summer camp for high school students, to local events and programmes based on the expertise and experience present on site, spanning from the development of new detectors for outreach activities to internships or competitions for students. Recently, a new national activity has been developed, focused on the preparation of interactive online materials for data analysis and its usage by students and teachers (see the contribution by C. Aramo in this conference).

The one aspect that all OCRA activities have in common is the exploitation of cosmic radiation for educational and outreach purposes. Cosmic radiation is easily intercepted and can be "made visible" through simple telescopes of particle detectors, so that it represents an excellent educational laboratory to introduce non-experts, especially students, to the study of physics and fundamental phenomena of nature.

In the following we will give an overview of the various activities offered by OCRA, with a particular focus on the 2020 online edition of the International Cosmic Day.

\section{Outreach activities organized by OCRA}

\subsection{Participation in International Cosmic Day}

The International Cosmic Day (ICD) [1] is an astroparticle physics outreach event for highschool students, bringing together students, schools, and various physics outreach projects from all over the world. Groups of scientists, teachers, and students meet for one day to learn about cosmic rays and perform an experiment with atmospheric muons. Usually, all participating groups investigate the same question: the zenith angle distribution of atmospheric muons. Using muon telescopes the students, with the help of the researchers, try to answer the following questions: is the number of air shower particles arriving from the horizon the same as from the zenith? What is the angular distribution of the cosmic muon flux? The students are enabled to work together like in an international collaboration, discussing their results in joint video conferences at the end of the day. In the end, they are invited to publish the results of their measurements in proceedings and receive a certificate of participation.

The ICD in 2020 took place on November 4, right in the middle of the second wave of the Covid-19 pandemic. OCRA thus decided to organize an online event accessible also for students that could not be at school but were home schooling at the time. 


\subsubsection{Setting up the online event}

Several challenges had to be overcome in order to offer a pleasant experience to the students. The most important question was how to make the students part of the experience in an active manner even if not present physically. The hands-on experience is the core of the traditional ICD and we wanted to preserve this feature also in the remote edition. The answer was the use of the Cosmic Ray Cube (CRC) [2]: this telescope is composed of 4 double-plane horizontal scintillation levels, orthogonal to each other. The special feature of this muon tracking system that made it particularly suitable to the remote edition of the ICD is the fact that it can be accessed not only by a dedicated computer software but also via a smartphone application that allows to observe the 3D event display of the telescope and to count the events registered by the CRC. The app, Cosmic Rays Live, can be downloaded from both Google Play and Apple Store [3] and allowed to involve students directly in the measurement process even during the remote event.

Another challenge that had to be overcome was the selection of the platform used to transmit the event and the technical setup needed to assure a good quality of sound and video. This led to the choice of the INFN Laboratori Nazionali del Gran Sasso (LNGS) and the Gran Sasso Science Institute (GSSI) in the close-by L'Aquila as the locations. The measurements were carried out at LNGS, where the local multimedia team was available to record the activities, while the event was directed and transmitted from GSSI that had the necessary access to the online platforms. The first choice was to use a Zoom webinar to transmit the event, given the good implementation of ways to interact with the students via the "Q\&A" tool and the easy access. However, almost 3000 students from more than 70 schools all over Italy signed up for the event and since most of them would follow from home, access needed to be guaranteed for all of them, which could not be achieved with the limit of 500 participants imposed by the Zoom license we had available. We therefore provided access via Zoom and a live stream on Youtube in parallel.

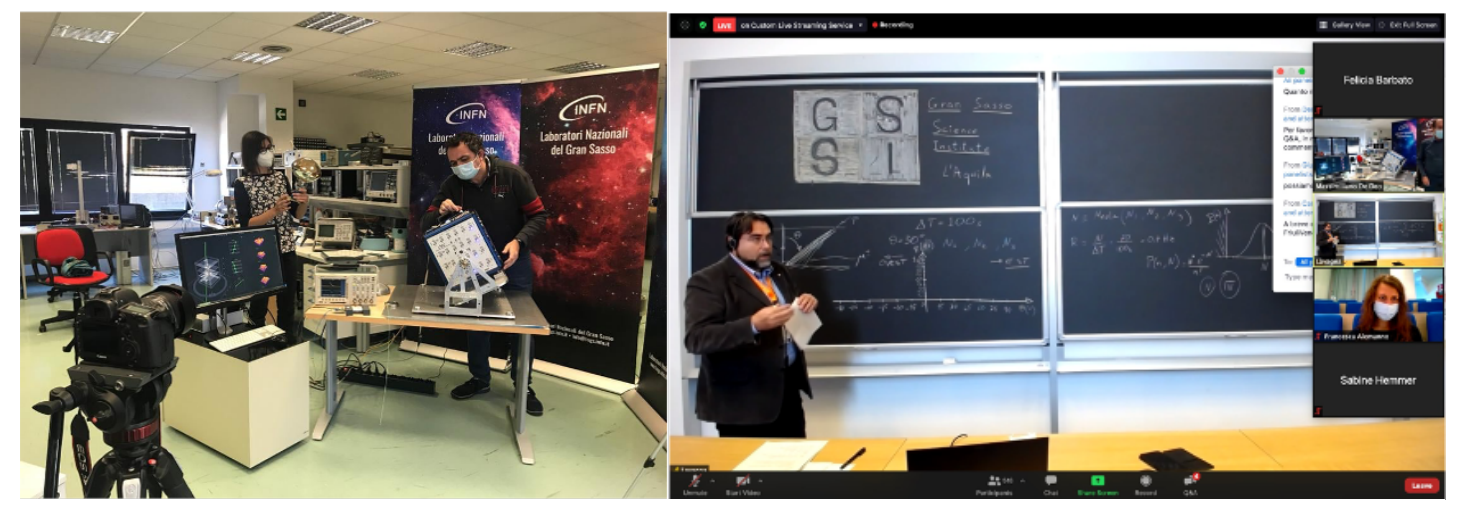

Figure 1: Activities during the OCRA ICD 2020.

\subsubsection{The program}

The OCRA ICD was scheduled to last three hours in the morning of November 4. After the institutional greetings, a short introduction to the physics of cosmic rays was given. This was deliberately kept short to have more time for the practical part of the event. In fact, many students had already followed introductory lectures organized online by the local OCRA groups in the previous days. A short presentation of OCRA and its activities followed before we dived into the core activities: the CRC and its app were explained to the students and then the measurement 
campaign started. For every angle the flux was measured and a group of students was assigned to the data taking and communicated the measurement results to the researchers via the chat. The data taking session was intertwined with short presentations by our researchers, a visit to the LNGS underground laboratory and the analysis and discussion of the data. A Kahoot quiz [4] gave the students the possibility to show what they had learned. The event closed with a connection to a researcher situated at the MAGIC telescopes in La Palma. To assure smooth transitions between the interventions, the event was moderated by a researcher experienced in conducting TV shows and outreach events. Over the course of the event, participants were invited to ask questions on our social profiles (instagram, facebook and twitter) that were answered in real time by short videos by our researchers.

In the afternoon or the days following the event, several OCRA groups organized online events aimed at data analysis and deepening of the concepts presented during the ICD with local schools.

\subsubsection{Evaluation}

The satisfaction of the participants of the OCRA ICD event was explored via two short questionnaires, one for the students and one for the teachers, with the invitation to rate several aspects from 1 (not true at all) to 5 (fully agree). A summary of all 1078 answers given by the students is shown in Fig. 2. As can be seen, general satisfaction was rather high, with an average vote of 3.9, and the event corresponded well to the expectations of the students (average vote 3.8). The contents were interesting and well presented (both rated 4.0) and the students were rather satisfied with the organization, with an average vote of 3.8. The question that received the lowest rating with 3.2 was the one investigating if the prior knowledge of the students was sufficient to follow the event. This is in agreement with similar polls carried out for the traditional ICD in the past. A total of $80 \%$ of all participating students said that they would recommend the event to their fellow students. No significant differences were observed between different groups of participants, such as different grades or different types of school.

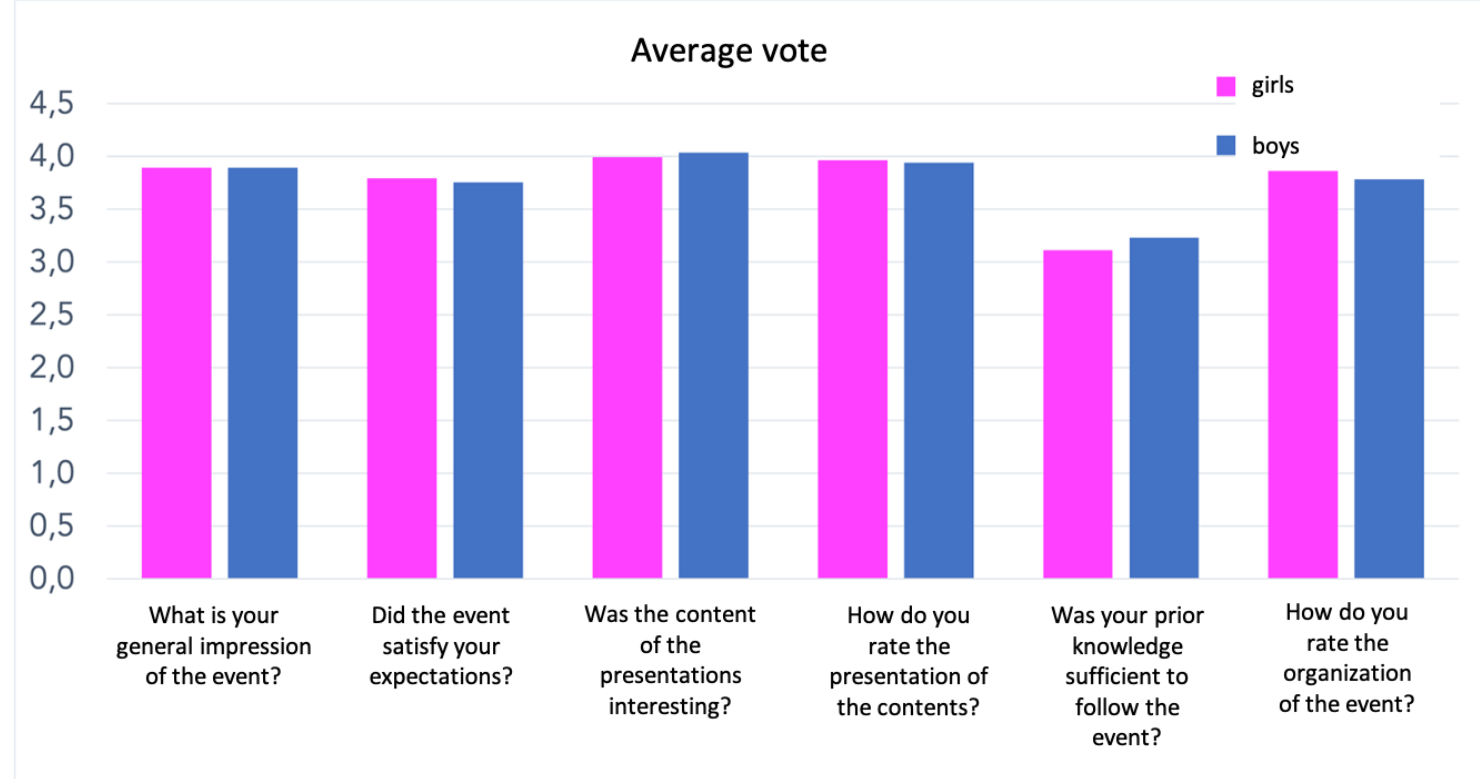

Figure 2: OCRA ICD student questionnaires. 


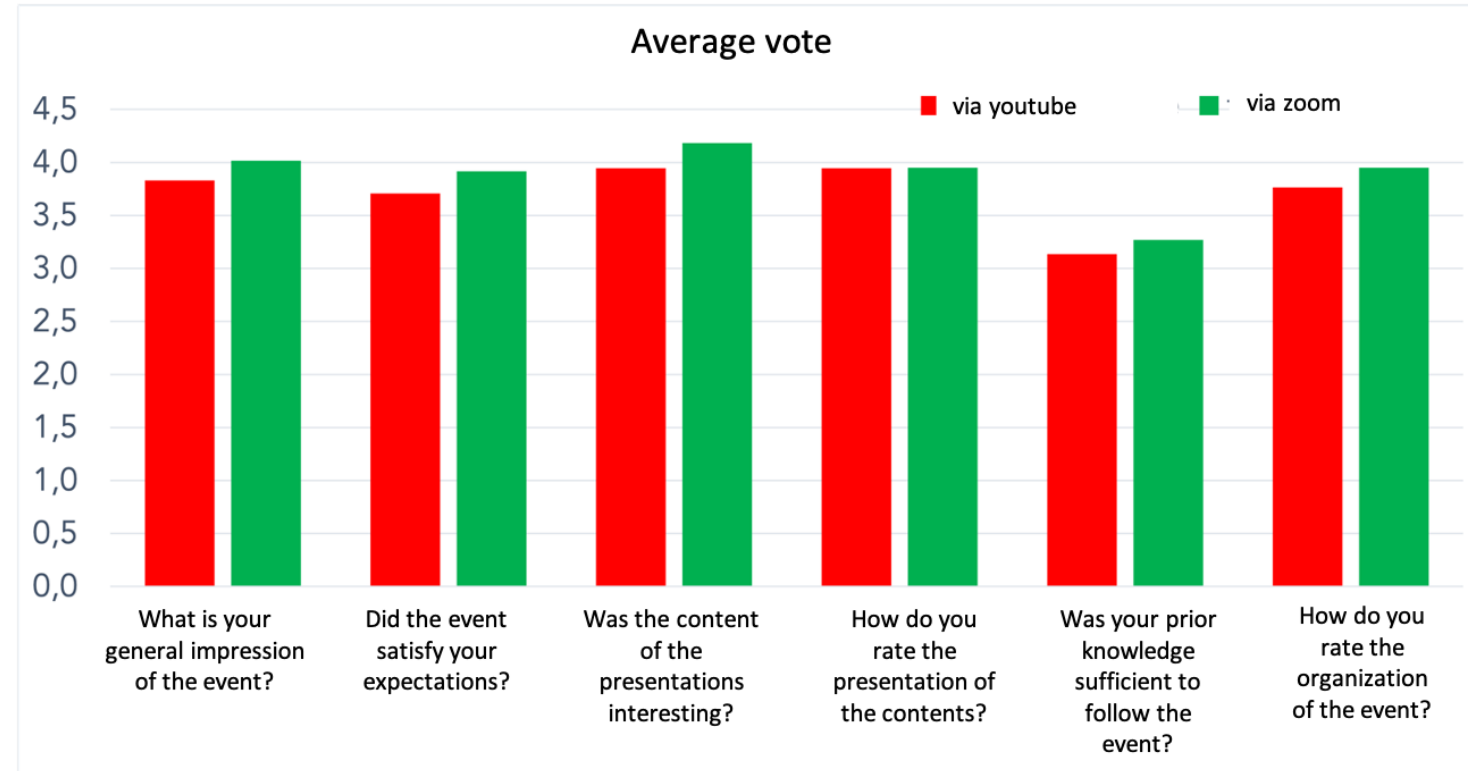

Figure 3: OCRA ICD questionnaires for students who participated via Youtube or via Zoom.

Figure 3 shows that the average votes were consistently slightly lower for participants that followed the event via Youtube compared to the votes expressed by the students that were able to access via Zoom on a first come-first serve basis. This difference in satisfaction can be explained by two main points. Firstly, on the Zoom platform the students were able to interact via the "Q\&A" tool and the chat. On Youtube there is no such tool as "Q\&A" and the chat was soon flooded with comments that did not allow for a proper use of this tool. Therefore, for those students that participated via Youtube, there was no direct way to interact with the researchers. Secondly, the quality of the co-viewing experience on Youtube gets impacted by the time delay variance of the streaming transmission. This means that the participation in activities like the quiz, but also in the measurement campaign, was less satisfying for the students attending the event on this channel.

When asked for their favourite part of the activities, the students gave very different answers. Many expressed their satisfaction about the fact that they had the chance to personally carry out the measurements, others enjoyed most the theoretical introduction on cosmic rays or the virtual visit to the underground laboratory or the MAGIC telescopes. Also, the interactive quiz was very appreciated by the students. The multitude of different answers on this question indicates that the program was well balanced in terms of diversity, offering something of interest for all tastes and inclinations.

The answers to the question of ways to improve the event covered technical aspects, as the quality of sound and image, as well as practical concerns like the density of activities without breaks and the duration of the event as well as the need to find a way to provide all students with the possibility to interact directly with the researchers without time delays.

The teacher questionnaires, depicted in Fig. 4, showed similar results as those of the students, with the general satisfaction even higher with an average rating of 4.2. Of the 98 teachers that answered the questionnaire, 88 would like to participate again with their students in the following year. The comments were predominantly positive, with some organisational note regarding the lack of sufficient breaks and the length of the event. 


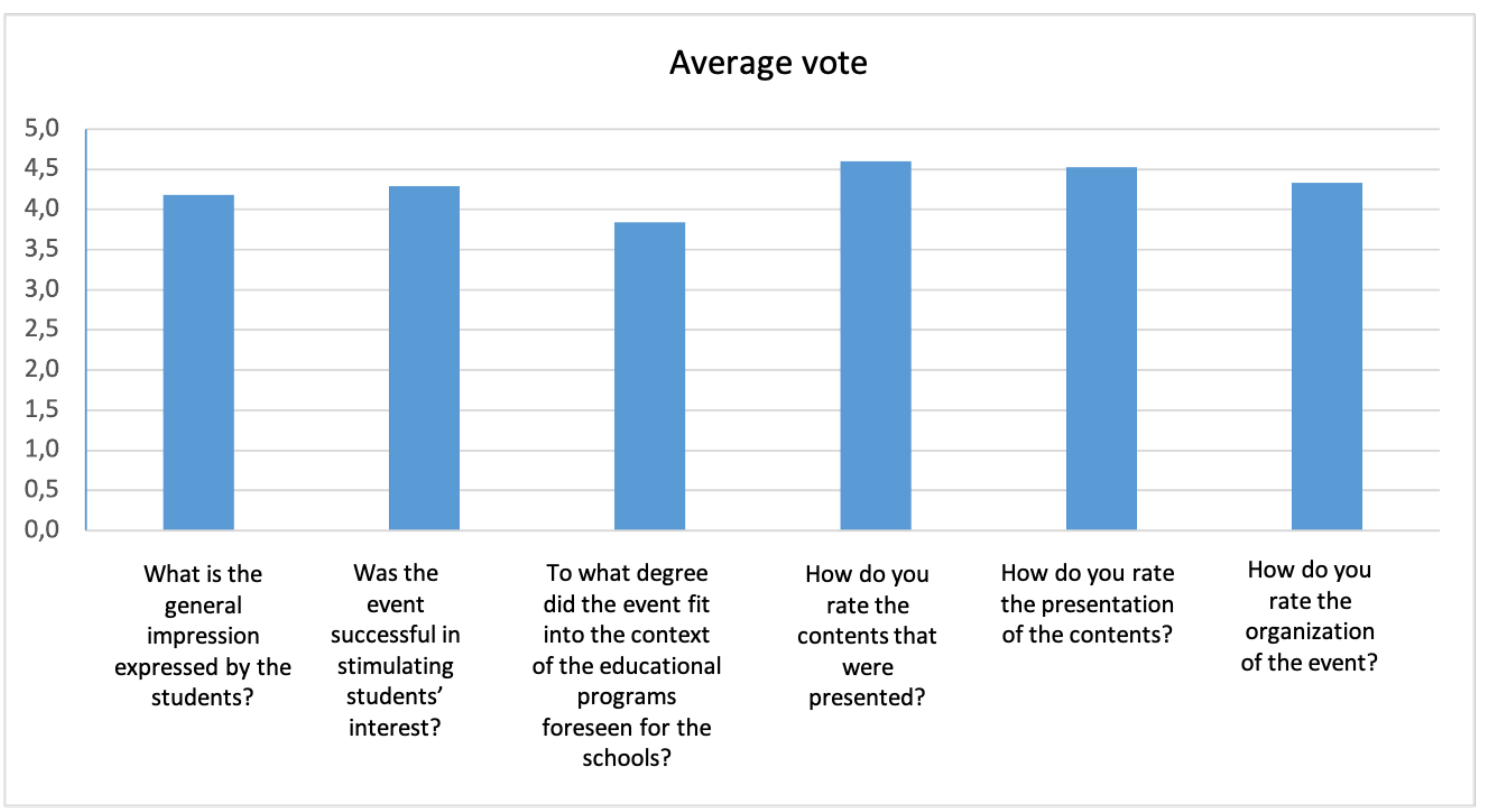

Figure 4: OCRA ICD teacher questionnaires.

\subsection{Science Camp for students}

Another milestone in the activities of the OCRA project is the yearly science camp for students. Two students from each local unit participating in the ICD are selected to join the camp which takes place in the spring of each year. The first camp took place in 2019 at the Gran Sasso with the participation of more than 30 students from all over Italy. The measurements carried out with the students aimed at determining the dependence of the cosmic ray flux on the altitude by means of a set of measurements in several locations around the Gran Sasso massif in central Italy [2].

In 2020, the science camp was supposed to take place at the INFN Laboratori Nazionali di Frascati (LNF) near Rome with an activity already tested and validated by the Rome group, i.e., the launching of a balloon with instrumentation for the measurement of cosmic rays (see the contribution by V. Bocci in this conference). Unfortunately, just a few weeks before the camp was supposed to take place, the Covid19 pandemic hit Italy and the camp had to be put on halt. We hope to be able to realize it in the spring of 2022.

\subsection{Online resources and teacher training}

During the first lockdown in Italy due to Covid19, in the spring of 2020, a series of online tools and interactive laboratories were developed and made available online on the website of the OCRA collaboration [5]. The original reason was to support high school students in their final school year with the preparation of their thesis work needed for the final exams. In parallel, materials for teachers were added with the scope of helping them in integrating the provided materials into their work in the classroom. Finally, an online course for teachers was organized in the spring of 2021 with an introduction to cosmic ray physics and a hands-on approach in presenting all laboratories available on the website. For more information refer to [2]. 


\subsection{Local activities}

The fourth pillar of the OCRA project are the local activities organized in an independent manner by the 21 units composing OCRA. These depend directly on the local expertise and manpower. In 2021, for instance, they comprised a locally organized online ICD event by the Lecce group carried out in parallel to the national one to accommodate the enormous demand by the schools that this group had received. Other groups offered lectures and seminars on cosmic rays for local schools on various subjects. Also, the development of muon telescopes for outreach activities, often in collaboration with local schools is carried out by several of the local groups. Finally, many groups host school students for some days or weeks for the Italian program of orientation and development of transversal skills required for all high school students, the socalled PCTO [6]. Within these programs students are introduced to the local experimental activities and carry out measurements and data analyses. In Naples, a competition for high schools on several subjects is organized every year (see contribution by R. Colalillo in this conference).

\section{Conclusions}

Since its foundation in 2018, OCRA has been growing constantly and it is safe to say that it has reached its goal of providing a national framework to the many local outreach events on cosmic rays present in INFN. It has maintained its core activity, the participation to the ICD by all local groups, even during the pandemic. In addition, many new events and programs have been developed, such as the summer camp and, recently, the online activities and the teacher training. These new aspects, in particular the teacher training, will be further developed, with the idea of turning it into a hands-on course for teachers, providing them with all the necessary knowledge and confidence to offer the activities in their classrooms.

\section{Acknowledgements}

A special acknowledgement goes to the group of local researchers from LNGS, GSSI and University of L'Aquila who organized the OCRA ICD 2020 online event.

\section{References}

[1] https://icd.desy.de/

[2] C. Aramo and S. Hemmer for the OCRA collab., Outreach Cosmic Ray Activities (OCRA): a program of astroparticle physics outreach events for high-school students, in proceedings of ICRC2019 PoS (ICRC2019) 173.

[3] https://play.google.com/store/apps/details?id=com.DigitalComoedia.CosmicRaysLive\&hl=it\&gl=U S and https://apps.apple.com/it/app/cosmic-rays-live/id1312853002

[4] https://kahoot.com/

[5] https://web.infn.it/OCRA/

[6] https://www.miur.gov.it/web/guest//linee-guida-dei-percorsi-per-le-competenze-trasversali-e-per-1orientamento 


\section{Full Authors List: OCRA Collaboration}

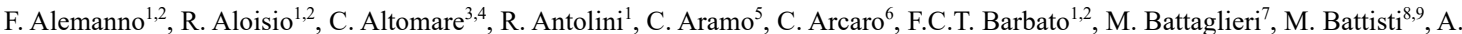
Bau $^{10,11}$, V.E. Bellinzona ${ }^{12}$, P. Bernardini ${ }^{13,14}$, A. Bersani ${ }^{7}$, M. Bertaina ${ }^{8,9}$, A. Berti8, B. Bertucci ${ }^{15,16}$, F. Bisconti ${ }^{8,9}$, E. Bissaldi ${ }^{3,4}$, V. Bocci $^{17}$, M. Boezio ${ }^{18,19}$, D. Boncioli ${ }^{1,20}$, M. Bondi ${ }^{7}$, L. Bonechi ${ }^{21}$, R. Bonino ${ }^{8,9}$, G. Bonnoli ${ }^{22}$, V. Bonvicini ${ }^{18}$, E. Bossini ${ }^{22,23}$, B.

Bottino $^{7,24}$, M. Buscemi2 ${ }^{25}$, B. Caccianiga ${ }^{26}$, L. Caccianiga ${ }^{26}$, A. Candela ${ }^{1}$, A. Capone ${ }^{17,27}$, M. Cariello ${ }^{7}$, R. Caruso ${ }^{25,28}$, G. Cataldi ${ }^{13}$, G Chiodi $^{17}$, G. Chiodini ${ }^{13}$, R. Colalillo, ${ }^{5,29}$, M.R. Coluccia ${ }^{13}$, F. Convenga ${ }^{13,14}$, S. Copello ${ }^{7,30}$, M. Corosu ${ }^{7}$, D. D’Urso ${ }^{31,32}$, F. Dal Corso ${ }^{6}$, S. Davini ${ }^{7}$, M. De Deo ${ }^{1}$, S. De Gateano ${ }^{3,4}$, M. De Laurentis ${ }^{5,29}$, I. De Mitri ${ }^{1,2}$, F. De Palma ${ }^{13,14}$, E. De Vito ${ }^{13,14}$, D. Dell'Aquila ${ }^{31,32}$, D. Depaoli $^{8,9}$, A. Di Luca ${ }^{12}$, F. Di Pierro ${ }^{8}$, B. Di Ruzza ${ }^{12}$, M. Di Santo ${ }^{1,2}$, G. Di Sciascio ${ }^{33}$, L. Di Venere, ${ }^{3,4}$, F. Dimiccoli ${ }^{12}$, K.

Dimitrios $^{1,2}$, F. Donnini ${ }^{15}$, M. Doro ${ }^{6,34}$, M. Duranti ${ }^{15}$, C. Evoli ${ }^{1,2}$, F. Fenu ${ }^{8}$, F. Fontanelli ${ }^{7,30}$, P. Fusco ${ }^{3,4}$, F. Gargano ${ }^{3}$, M. Gervasi ${ }^{10,11}$, A. Giampaoli ${ }^{1}$, N. Giglietto ${ }^{3,4}$, F. Giordano ${ }^{3,4}$, I. Gnesi $^{35,36}$, S. Gonzi ${ }^{21}$, D. Grandi ${ }^{10,11}$, M. Graziani ${ }^{15,16}$, S. Hemmer ${ }^{6}$, F. Iacoangeli ${ }^{17}$, A. Insolia ${ }^{25,28}$, S. Iovenitti $3^{26,37}$, V. Ippolito ${ }^{17}$, G. La Vacca ${ }^{10,11}$, G. La Verde ${ }^{5,29}$, E. Leonora ${ }^{25}$, S. Levorato ${ }^{18}$, D. Liguori ${ }^{35,38}$, P. Lipari ${ }^{17}$, F. Longo ${ }^{18,39}$, F. Loparco ${ }^{3,4}$, R. Lopez Coto $^{6}$, S. Loporchio ${ }^{3}$, A. Marino ${ }^{5,40}$, G. Marsella ${ }^{25,41}$, D. Martello ${ }^{13,14}$, M.N. Mazziotta ${ }^{3}$, A. Menegolli ${ }^{42,43}$, S. Miozzi ${ }^{33}$, H. Miyamoto ${ }^{8}$, E. Mocchiutti ${ }^{18}$, S. Morganti ${ }^{17}$, F. Morsani ${ }^{22}$, R. Munini ${ }^{18,19}$, R. Mussa ${ }^{8}$, F. Nozzoli ${ }^{12}$, A. Nucita $^{13,14}$, G.C. Organtini ${ }^{17,27}$, G. Ottonello ${ }^{7}$, F. Pantaleo ${ }^{3,4}$, R. Paoletti ${ }^{22}$, F. Parodi ${ }^{7}$, L. Perrone ${ }^{13,14}$, L. Pesenti ${ }^{10,11}$, S. Petrera ${ }^{12,}$, C.

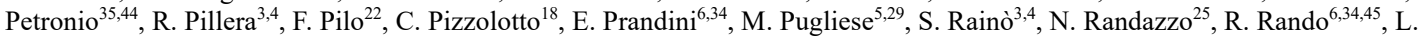
Recchia $^{17}$, E. Ricci ${ }^{12}$, M. Rinaudo ${ }^{8,9}$, V. Rizi ${ }^{1,20}$, N. Rossi ${ }^{1}$, D. Rozza ${ }^{31,32}$,F. Salamida ${ }^{1,20}$, P. Savina ${ }^{13,14}$, V. Scherini ${ }^{13,14}$, M.

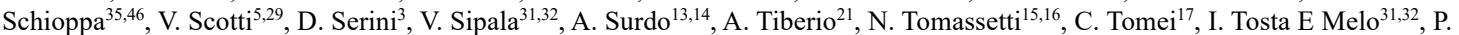
Turco $^{35,46}$, E. Vannuccini ${ }^{21}$, V. Vecchiotti ${ }^{1,2}$, I. Veronesi ${ }^{5,47}$, G. Zampa ${ }^{18}$

${ }^{1}$ INFN Laboratori Nazionali del Gran Sasso, Assergi, Italy

${ }^{2}$ Gran Sasso Science Institute, L’Aquila, Italy

${ }^{3}$ INFN Sezione di Bari, Bari, Italy

${ }^{4}$ Dipartimento Interateneo di Fisica "M. Merlin", University and Politecnico of Bari, Bari, Italy

${ }^{5}$ INFN Sezione di Napoli, Naples, Italy

${ }^{6}$ INFN Sezione di Padova, Padua, Italy

${ }^{7}$ INFN Sezione di Genova, Genua, Italy

${ }^{8}$ INFN Sezione di Torino, Turin, Italy

${ }^{9}$ Dipartimento di Fisica, University of Turin, Turin, Italy

${ }^{10}$ INFN Sezione di Milano Bicocca, Milan, Italy

${ }^{11}$ Dipartimento di Fisica "Giuseppe Occhialini", University of Milan-Bicocca, Milan, Italy

${ }^{12}$ Trento Institute for Fundamental Physics and Applications, Trento, Italy

${ }^{13}$ INFN Sezione di Lecce, Lecce, Italy

${ }^{14}$ Dipartimento di Matematica e Fisica "Ennio de Giorgi”, University of Salento, Lecce, Italy

${ }^{15}$ INFN Sezione di Perugia, Perugia, Italy

${ }^{16}$ Dipartimento di Fisica e Geologia, University of Perugia, Perugia, Italy

${ }^{17}$ INFN Sezione di Roma I, Rome, Italy

${ }^{18}$ INFN Sezione di Trieste, Trieste, Italy

${ }^{19}$ Institute for Fundamental Physics of the Universe, Trieste, Italy

${ }^{20}$ Dipartimento di Scienze Fisiche e Chimiche, University of L'Aquila, L'Aquila, Italy

${ }^{21}$ INFN Sezione di Firenze, Florence, Italy

${ }^{22}$ INFN Sezione di Pisa, Pisa, Italy

${ }^{23}$ Dipartimento di Fisica, University of Pisa, Pisa, Italy

${ }^{24}$ Physics Department, Princeton University, Princeton, USA

${ }^{25}$ INFN Sezione di Catania, Catania, Italy 
${ }^{26}$ INFN Sezione di Milano, Milan, Italy

${ }^{27}$ Dipartimento di Fisica, University La Sapienza, Rome, Italy

${ }^{28}$ Dipartimento di Fisica e Astronomia “E. Majorana”, University of Catania, Catania, Italy

${ }^{29}$ Dipartimento di Fisica "Ettore Pancini”, University of Naples "Federico II", Naples, Italy

${ }^{30}$ Dipartimento di Fisica, University of Genua, Genua, Italy

${ }^{31}$ INFN Laboratori Nazionali del Sud, Catania, Italy

${ }^{32}$ Dipartimento di Chimica e Farmacia, University of Sassari, Sassari, Italy

${ }^{33}$ INFN Sezione di Roma Tor Vergata, Roma, Italy

${ }^{34}$ Dipartimento di Fisica e Astronomia “G. Galilei”, University of Padua, Padua, Italy

${ }^{35}$ INFN Laboratori Nazionali di Frascati, Gruppo Collegato di Cosenza, Cosenza, Italy

${ }^{36}$ Centro Ricerche Enrico Fermi, Rome, Italy

${ }^{37}$ Dipartimento di Fisica “A. Pontremoli”, University of Milan, Milan, Italy

${ }^{38}$ Liceo Patrizi, Cariati, Italy

${ }^{39}$ Dipartimento di Fisica, University of Trieste, Trieste, Italy

${ }^{40} \mathrm{CNR}$ - Istituto di Scienze Applicate e Sistemi Intelligenti “Eduardo Caianiello", Pozzuoli, Italy

${ }^{41}$ Dipartimento di Fisica e Chimica “Emilio Segrè”, University of Palermo, Palermo, Italy

${ }^{42}$ INFN Sezione di Pavia, Pavia, Italy

${ }^{43}$ Dipartimento di Fisica, University of Pavia, Pavia, Italy

${ }^{44}$ Liceo Volta, Reggio Calabria, Italy

${ }^{45}$ Centro di Ateneo di Studi e Attività Spaziali “G. Colombo”, University of Padua, Padua, Italy

${ }^{46}$ Dipartimento di Fisica, University of Calabria, Arcavacata di Rende, Italy

${ }^{47}$ Dipartimento di Matematica, University of Salerno, Salerno, Italy 